\section{$\underset{\substack{\text { hommes } \\ \text { \& migrations }}}{ }$}

\section{Hommes \& migrations}

Revue française de référence sur les dynamiques

migratoires

1317-1318 | 2017

L'Europe en mouvement

\title{
Chauvinisme frontalier sur la « route des Balkans »
}

«Crise » migratoire et relations interétatiques depuis l'été 2015

\section{Morgane Dujmovic et Pierre Sintès}

\section{(2) OpenEdition \\ 1 Journals}

Édition électronique

URL : http://journals.openedition.org/hommesmigrations/3893

DOI : 10.4000/hommesmigrations.3893

ISSN : 2262-3353

Éditeur

Musée national de l'histoire de l'immigration

Édition imprimée

Date de publication : 1 avril 2017

Pagination : 107-115

ISBN : 978-2-919040-38-4

ISSN : 1142-852X

Référence électronique

Morgane Dujmovic et Pierre Sintès, "Chauvinisme frontalier sur la "route des Balkans » ", Hommes \& migrations [En ligne], 1317-1318 | 2017, mis en ligne le 01 avril 2020, consulté le 04 janvier 2020. URL: http://journals.openedition.org/hommesmigrations/3893; DOI : 10.4000/hommesmigrations.3893 


\title{
CHAUVINISME FRONTALIER SUR LA 《ROUTE DES BALKANS 》 «CRISE » MIGRATOIRE ET RELATIONS INTERÉTATIQUES DEPUIS L'ÉTÉ 2015
}

Par Morgane DUJMOVIC, doctorante contractuelle, ATER en géographie, Telemme AMU-CNRS, et Pierre SINTĖS, maître de conférences en géographie, Telemme AMU-CNRS.

\author{
Traversés de points de passage aléatoires et de frontières \\ fermées et militarisées, les pays des Balkans se trouvent \\ au cœur de la route que tentaient d'emprunter les réfugiés \\ durant la crise migratoire de 2015. Les États balkaniques \\ ont géré leur position géographique stratégique entre une \\ adaptation aux règles de la coopération migratoire européenne \\ et le repli sur des logiques nationalistes et exclusives. Résultat, \\ I'espace de transit balkanique représente un nouveau limes, \\ une frontière extérieure, lointaine et mouvante, désormais \\ quasi infranchissable. Multipliant les murs, cette zone-tampon \\ est devenue le repoussoir de l'Europe.
}

L'instabilité politique et sociale qui touche le Proche et le Moyen-Orient, ainsi que d'autres pays d'Asie et d'Afrique, a alimenté depuis plusieurs décennies des flux d'individus migrant vers l'Europe ${ }^{1}$. Ceux-ci ont été décuplés à partir de l'été 2015 en raison de la dramatisation des conflits dans certains de ces pays (notamment la Syrie, l'Irak et l'Afghanistan). Le renforcement du contrôle frontalier en Méditerranée occidentale et centrale a réorienté les parcours migratoires vers la péninsule balkanique où 500000 migrants auraient transité au plus fort de la période dite de " crise » des migrants (septembre-décembre 2015) ${ }^{2}$. Cette sous-région est ainsi devenue la principale porte d'entrée vers l'espace Schengen, faisant de la question migratoire dans les Balkans un enjeu continental. Depuis la chute des régimes communistes, la position de cet ensemble comme zone pivot a justifié les discours et les politiques de l'Union européenne (UE), incitant les États balkaniques à s'impliquer dans la gestion intégrée des questions migratoires mise en œuvre à l'échelle continentale. 
Les conditionnalités européennes se sont concrétisées avec le processus de stabilisation et d'association (PSA) lancé en 1999 et sa déclinaison en accords de stabilisation et d'association (ASA). Ces derniers ont constitué les cadres politiques bilatéraux des négociations d'adhésion pour les pays des «Balkans occidentaux ». Les autorités des pays alors candidats ou candidats potentiels de la région (Albanie, Croatie, Bosnie-Herzégovine, Serbie, Monténégro, République de Macédoine, et depuis peu Kosovo) ont ainsi dû adapter leurs normes et pratiques de contrôle des migrants non européens pour se voir eux-mêmes inscrits progressivement sur la " liste blanche » des visas Schengen qui permet à leurs propres ressortissants de voyager plus librement en Europe.

Cette décennie de collaboration explique certaines continuités dans les réponses apportées par les autorités des Balkans à la « crise » de l'été 2015. L'instrument d'aide de préadhésion (IAP), qui finance depuis 2007 les réalisations européennes dans la région, a ainsi été utilisé pour des mesures de "gestion de la crise migratoire ». Certaines infrastructures issues de ce programme ont été réemployées pour l'hébergement de milliers d'individus (points-frontières, centres « d'accueil » et « de rétention » modernisés). Face à ce phénomène inédit et massif, un corridor migratoire a été mis en place pour permettre le franchissement officiel des frontières du nord de la Grèce à l'Allemagne sans être muni de visa (septembre 2015-mars 2016). Présenté comme une réponse humanitaire permettant de sécuriser les parcours, ce mécanisme de gestion migratoire commune n'a pourtant pas résisté aux mesures des États qui ont privilégié une sécurisation de leurs territoires. Au cours de cette période, les relations entre États balkaniques ont oscillé entre coopération et logiques nationales, imposant sur le terrain un nouveau chapitre brûlant pour des relations interétatiques déjà tendues. L'exemple grec montre comment cette séquence est allée de pair avec l'émergence (ou le retour) d'un discours sur la protection des frontières nationales, adossé à un argumentaire sur l'héritage de leur formation géopolitique. L'épisode de 2015 semble avoir éloigné les protagonistes d'un cadre véritablement coopératif, pour les faire renouer avec une rhétorique chauvine, voire nationaliste, aux dépens des mécanismes basés sur la solidarité entre États européens.

\section{La « route des Balkans », entre coopération et tensions aux frontières}

Depuis les années 1990, des routes migratoires au tracé changeant traversent les différents pays de la péninsule balkanique. Elles sont empruntées par des migrants " irréguliers » comme par de potentiels demandeurs d'asile originaires principalement d'Afrique, d'Asie et du MoyenOrient. Malgré le développement discret de tels flux depuis une vingtaine d'années, aucun État des Balkans n'était préparé aux arrivées massives qui ont débuté à l'été 2015. C'est alors que culmine réellement la fonction "d'espace de transit " associée à l'espace balkanique depuis les années 2000. Plusieurs gouvernements ont, tout d'abord, répondu en adoptant des mesures unilatérales de protection de leur territoire. Dès le 20 août 2015, les autorités macédoniennes proclament l'état d'urgence, qui permet le renforcement des effectifs policiers et militaires sur les frontières du pays. Cette mesure de contrôle est justifiée par le gouvernement qui décrit « une situation alarmante en raison d'une pression accrue à la frontière Sud » due aux "passages illégaux massifs en provenance de la Grèce ${ }^{3}$ ». Le 15 septembre, la Hongrie donne le ton en achevant l'édification d'une clôture de barbelés à sa frontière avec la Serbie ${ }^{4}$. S'ensuivent 
des heurts violents, comme le 17 septembre au poste-frontière de Horgoš où les policiers hongrois font usage de gaz lacrymogènes et de canons à eau. De façon presque immédiate, ce dispositif entraîne des contournements : le soir même, un nouveau point de passage s'ouvre entre la ville de Šid en Serbie et le village de Tovarnik en Croatie. Le 16 septembre, plusieurs dizaines de migrants franchissent illégalement la frontière de la Croatie ; le 17, on dénombre plus de 6000 entrées à ŠidTovarnik. «Massif» et «incontrôlé », selon les mots de la présidente croate Kolinda Grabar Kitarović, ce phénomène entraîne rapidement la crispation des autorités croates qui ferment le lendemain la quasi-totalité des points-frontières avec la Serbie ${ }^{5}$. Les réactions serbes ne se font pas attendre: en représailles, les véhicules de transports de marchandises croates sont interdits sur le territoire serbe et les importations croates sont bloquées. La crise entre les deux pays est néanmoins de courte durée puisque les autorités croates décident la réouverture des points-frontières le 26 septembre. Cet exemple consécutif à la " crise " migratoire est emblématique de la réactivation de tensions entre voisins, ici anciens ennemis du temps des guerres ex-yougoslaves (1991-1995), aujourd'hui membre et candidat à l'UE. Il signale d'emblée que de tels héritages ont pu complexifier plus ou moins discrètement la gestion des arrivées et de la circulation à la frontière extérieure de l'UE au cours de cette période.

Face à l'ampleur du phénomène migratoire de l'été 2015, les premières réactions unilatérales ont pourtant vite donné lieu à une coopération de circonstance avec la mise en place progressive d'un corridor migratoire officiel, du sud de la République de Macédoine à l'Autriche. Lors d'une réunion extraordinaire à Bruxelles le 25 octobre 2015 entre les différents chefs de gouvernement des Balkans et des États membres de l'UE, JeanClaude Juncker promeut un dispositif censé permettre "un mouvement graduel, contrôlé et ordonné des personnes sur la route des Balkans occidentaux $^{6}$ ». Dans son ensemble, le corridor encadré par les autorités des différents États - en particulier la République de Macédoine, la Serbie, la Croatie et la Slovénie - remplit trois fonctions officielles : la protection et la sécurisation du passage des migrants face aux dangers du voyage, l'organisation rapide du transit vers l'Allemagne ou l'Autriche, et le contrôle des groupes de migrants pour prévenir leur éventuelle dispersion. Deux activités essentielles sont menées le long de ce parcours : l'enregistrement systématique des migrants et la distribution d'aide humanitaire.

\section{Du corridor aux verrous intra-européens}

Très vite pourtant, alors que ce corridor se structure et que les arrivées continuent en mer Égée, on assiste à un véritable jeu de frontières marqué par des fermetures successives. C'est, en premier lieu, entre États membres voire à l'intérieur même de l'espace Schengen que des contrôles sont restaurés et que des barrières sont érigées. Dès le 18 septembre, la Hongrie continue de se claquemurer en installant une clôture haute de 3,5 mètres sur les 41 kilomètres de frontière avec la Croatie qui ne sont pas séparés par la Drave. Le Premier ministre Viktor Orbán déclare alors que les autorités se Face à l'ampleur du phénomène migratoire de l'été 2015 , les premières réactions unilatérales ont pourtant vite donné lieu à une coopération de circonstance avec la mise en place progressive d'un corridor migratoire officiel, du sud de la République de Macédoine à l'Autriche. préparent à faire de même aux frontières avec la Slovénie et la Roumanie. La fermeture effective de la frontière HongrieCroatie le 16 octobre à minuit modifie l'itinéraire du corridor vers la Slovénie, entraînant la réaction immédiate du ministre slovène des Affaires 
étrangères Karl Erjevec qui pose une limite à cette collaboration en annonçant le 19 octobre que Ljubljana n'acceptera que 2500 migrants par jour. Alors que le parlement slovène valide l'envoi de l'armée sur les frontières, les migrants continuent d'affluer à Preševo, dans le Sud de la Serbie, créant un engorgement de près de 10000 personnes. La fermeture des frontières n'est pas l'apanage des États du Sud-Est européen puisque l'Autriche annonce l'érection d'une barrière à sa frontière avec la Slovénie le 28 octobre, alors même que plusieurs milliers de personnes
Des fer metures temporaires

de postes-frontières à

l'érection de clôtures

physiques, la mise en place

de ces différents verrous

révèle une politique du

«chacun pour soi » qui puise

dans la crainte d'une décision

allemande ou autrichienne

de mettre fin à leur politique

d'accueil. attendent de passer le poste de Šentilj-Spielfeld. C'est en réaction à cette décision que la Slovénie entreprend à partir du 10 novembre de clôturer sa frontière avec la Croatie le long de la rivière Sotla, puis de la Kupa. Les travaux de clôture menés par l'Autriche au postefrontière de Spielfeld débutent le 7 décembre : le grillage de 3,7 kilomètres de long et 2,5 mètres de haut est le premier mur jamais érigé entre deux pays de la zone Schengen. De l'autre côté du corridor, un autre loquet se met en place au sud de la République de Macédoine où un grillage est installé fin novembre.

Des fermetures temporaires de postes-frontières à l'érection de clôtures physiques, la mise en place de ces différents verrous révèle une politique du « chacun pour soi » qui puise dans la crainte d'une décision allemande ou autrichienne de mettre fin à leur politique d'accueil. Comme cela a pu être constaté pour les périodes précédentes ${ }^{7}$, ces décisions en aval des routes migratoires agissent par effet rebond sur l'ensemble du parcours balkanique. Ce dispositif ambivalent, conjuguant transit vers l'Ouest et blocages, apparait clairement dans les politiques nationales consistant à imposer par à-coups des quotas journaliers pour juguler les flux en fonction des décisions du voisin. Les restrictions chiffrées de l'Autriche se répercutent sur tous les chaînons des parcours balkaniques jusqu'à la frontière gréco-macédonienne où l'accès au corridor est réservé aux originaires de Syrie, d'Irak et d'Afghanistan (novembre 2015), puis seulement aux personnes venant de villes touchées directement par la guerre (mars 2016). C'est finalement l'échec du sommet UE-Turquie du 7 mars 2016 qui sonne le glas du corridor balkanique : le 8 mars à minuit, la Slovénie ferme définitivement ses frontières, entraînant le blocage de toute la « route des Balkans ». Par effet domino, les frontières croates et serbes sont fermées le soir même, bloquant des milliers de migrants sur les différents points nodaux du corridor, pris au piège de ces politiques migratoires restrictives.

Les raisons qui incitent au départ restant nombreuses, le verrouillage du corridor n'a pas provoqué l'arrêt du phénomène migratoire. En revanche, le renforcement des contrôles frontaliers a provoqué une flambée des prix du passage : on estime que les traversées de la République de Macédoine et de la Serbie se monnayaient respectivement 1000 et 1500 euros fin avril 2016. Malgré la fermeture officielle du corridor, les routes des Balkans restent donc actives : plutôt que de se tarir, les flux semblent à présent se redéployer vers de nouveaux points de passage et de nouvelles routes plus dangereuses, maintenant ainsi la pression sur ce dossier dans les relations interétatiques.

\section{Chauvinisme et provocations militaires à la frontière gréco- turque}

En 2015, la Grèce est en position particulière puisqu'elle voit transiter sur son sol presque la totalité du flux des migrants : au moins 850000 personnes sont entrées sur le sol européen à partir de la frontière gréco-turque au cours de cette période. Mais, après la fermeture de la « route des 
Balkans » officielle, plus de 10000 personnes sont bloquées dans les îles de l'Égée, et 45000 sur le continent, laissant la Grèce dans une situation très tendue en matière d'accueil. Le premier effet du bouleversement de 2015 est le renforcement de l'internationalisation de la gestion migratoire en Grèce. Depuis plusieurs années déjà, les institutions européennes sont présentes sur le sol grec pour participer au traitement de la question migratoire ${ }^{8}$. L'agence Frontex a lancé depuis 2014 l'opération maritime Poséidon, consolidée une première fois en décembre 2015 par l'envoi de la force de réaction rapide (Rabit). Le dernier acte de ce dispositif repose sur l'accord entre l'UE et la Turquie qui permet, à partir du 4 avril 2016, le renvoi en Turquie de migrants depuis les îles de l'Égée. Alors que les trois premiers mois de 2016 laissaient attendre des chiffres élevés (80 000 entrées de janvier à mars), le nombre de passages se serait effondré si l'on en croit les rapports de Frontex qui font suite aux accords UE-Turquie (-90\% au mois d'avril $\left.{ }^{9}\right)$, même si des migrants continuent d'arriver chaque mois, en particulier sur les îles de Lesbos, Samos, Chios, Leros et Kos. D'un point de vue géopolitique, les implications de cet accord sont lourdes pour la Grèce : il s'agit d'une délégation de souveraineté significative puisque des migrants sont expulsés depuis son territoire en vertu d'une décision prise ailleurs en Europe, alors que les renvois, suspendus depuis 2013, reprennent depuis les autres États membres vers la Grèce ${ }^{10}$. Plus encore, les autorités grecques sont contraintes de tolérer la présence de fonctionnaires turcs en uniformes sur les îles de l'Égée (6 à Chios, 8 à Lesbos, 5 à Samos, 3 à Leros et 3 à Kos) et de considérer la Turquie comme un partenaire et un pays sûr pour le renvoi de migrants.

Or c'est une toute autre image qui est promue depuis des années par les autorités grecques pour justifier leur position au regard de deux questions géopolitiques qui la mettent aux prises avec le pays voisin : la question de Chypre ${ }^{11}$ et le différend sur les eaux territoriales de la mer Égée ${ }^{12}$, qui refont surface à la faveur de la « crise » migratoire. L'un des gardes-côtes envoyé par Ankara à Chios fin mars 2016 a ainsi été empêché de travailler car son passeport présentait un tampon d'entrée dans la République turque de Chypre du Nord, non reconnue par la Grèce ${ }^{13}$. Plus grave, depuis février 2016, des violations de l'espace aérien et des eaux territoriales grecques se sont multipliées. Fin avril, un garde-côte turc a même tenté de contraindre un pêcheur grec de le suivre dans les eaux territoriales turques pour y être arraisonné alors que ce dernier remontait ses filets dans les eaux grecques, provoquant l'intervention de la marine de guerre des deux pays ${ }^{14}$. Lors des dernières semaines de janvier 2017, des mouvements de troupes répétés autour de lî̀lot contesté d'Imia, mettant en scène le général en chef des forces armées turques Hulusi Akar ${ }^{15}$, faisaient ressurgir des tensions que l'on pensait oubliées, plus de 20 ans après les incidents qui avaient vu les deux pays frôler le conflit armé ${ }^{16}$. Cette tension

8. Laurence Pillant, Louise Tassin, « Lesbos, l'île aux grillages. Migrations et enfermement à la frontière gréco-turque ", in Cultures \& Conflits, $n^{\circ}$ 99-100, 2015, pp. 25-55 ; Laurence Pillant, «Introduction of procedures for migration controls in Greece. Case study in the Aegean Islands ", in Lisa Anteby-Yemini, Virginie Baby-Collin, Sylvie Mazzella, Stéphane Mourlane, Cédric Parizot, Céline Regnard, Pierre Sintès (dir.), Borders, Migrations and Mobilities. Perspectives from the Mediterranean, Berlin Peter Lang, 2014, pp. 71-85. 9. http://frontex.europa.eu/news/number-of-migrants-arriving-in-greece-dropped-9o-in-april-6e70Bw 10. http://www.lemonde.fr/europe/article/2016/12/og/les-refugies-de-nouveau-renvoyes-vers-la-grece_5046287_3214.html 11. La question chypriote oppose la Grèce et la Turquie depuis l'invasion par l'armée turque du Nord de l'île à l'été 1974. La partition qui en a résulté a abouti à la séparation des Chypriotes grecs (soutenus par la Grèce) et turcs (soutenus par la Turquie) dans deux États distincts. Elle est une source de tension diplomatique récurrente dans la région. 12. La frontière maritime entre la Grèce et la Turquie est le fruit de plusieurs accords internationaux (traité de Lausanne de 1923 et convention italo-turque d'Ankara en 1932). Un contentieux y porte sur l'extension des eaux territoriales, que la Grèce désirerait porter depuis 1974 à 12 miles nautiques (contre 6 miles à présent). Cette position a conduit Ankara à refuser de signer en 1982 la Convention des Nations Unies sur le droit à la mer qui appuie la revendication grecque (voir Georges Prévélakis, Géopolitique de la Grèce, Paris, Complexe, 1997, p. 27). 13. http://www.tovima.gr/society/article/?aid=786108 14. http://www.protothema. gr/greece/article/574924/ti-trehei-stis-oinousses-me-tous-tourkous/ 15. http://www.lemonde.fr/europe/article/2017/01/30/ provocation-turque-au-large-de-la-grece $50714693214 . \mathrm{html}$ 16. Le différend frontalier avait déjà amené les deux pays au bord du conflit armé autour de cet îlot en 1995-1996 alors que des journalistes turcs y avaient débarqué pour hisser le drapeau turc (voir Ekavi Athanassopoulou, "Blessing in disguise? The Imia crisis and turkish-greek relations ", in Mediterranean Politics, vol. 2, $n^{\circ} 3,1997$, pp. 76-101). 
semble culminer alors qu'une mission de l'Otan est déployée dans la région depuis février 2016 visant à limiter l'activité des passeurs à la frontière. La situation de tension entre les deux pays a cependant contraint le secrétaire général de l'organisation Jens Stoltenberg à préciser que «les forces de la Grèce et celles de la Turquie s'abstiendront d'opérer dans les eaux territoriales et l'espace aérien de l'autre pays ${ }^{17}$ ». Depuis le sommet de l'organisation du 8-9 juin 2016 à Varsovie, les autorités turques ont même demandé à ce que les opérations de surveillance de l'Otan ne concernent pas les zones des îles de Samos et de Kos sous le prétexte qu'il s'agirait de zones démilitarisées, faisant pression par ailleurs pour que soit mis un terme à cette mission.

\section{La migration au cœur des tensions entre la Grèce et ses voisins}

Au cours de cette période, le contexte migratoire et sa gestion semblent avoir été favorables au retour de rhétoriques nationalistes dans les pays de la région par l'utilisation régulière de la thématique de la maitrise et de l'intégrité des frontières nationales. Cela a notamment été le cas sur la frontière septentrionale de la Grèce autour de la question du camp de fortune d'Idoméni en mars 2016. Cet ensemble de tentes abritait à son maximum 10 à 12000 personnes bloquées par la fermeture progressive de la frontière par les autorités macédoniennes, en mars 2016. Dans les semaines et les mois qui suivent, la situation se complique et le quotidien sur le terrain est ponctué de heurts et de protestations : le 19 novembre 2015, 2000 migrants se couchent sur les voies ferrées; le 23 novembre, plusieurs migrants se cousent les lèvres; le 26 novembre et le 2 décembre, des échauffourées éclatent entre forces de l'ordre et migrants qui tentent de passer la frontière ; le 9 décembre, des centaines de policiers grecs font irruption dans le camp pour évacuer les migrants vers Athènes... Après les naufrages dans les îles, c'est à Idomeni que la crise humanitaire s'est transformée en " catastrophe humanitaire " selon le commissaire européen aux migrations, Dimitris Avramopoulos ${ }^{18}$. Par sa médiatisation, ce lieu est devenu très rapidement un symbole mondial du sort des réfugiés jusqu'au mois de mai 2016, quand les autorités grecques ont finalement évacué le camp dont la plupart des occupants ont été transférés vers des structures d'accueil dans la région.

Mais, pour une lecture complète de cet épisode, il est nécessaire d'évoquer l'héritage qui caractérise cette région. La frontière gréco-macédonienne a été depuis la Seconde Guerre mondiale l'un des lieux de matérialisation du rideau de fer, puis une frontière extérieure de l'espace Schengen autant qu'une aire de mise en place des programmes européens de coopération transfrontalière ${ }^{19}$. Elle est surtout, depuis les années 1990, un lieu d'affrontement autour de la question du nom contesté par la Grèce de la République de Macédoine qui fait de son côté usage de symboles que les autorités grecques tiennent pour purement helléniques ${ }^{20}$. Cette tension, qui dure depuis plusieurs décennies ${ }^{21}$ sur fond de présence d'un groupe slavophone en Grèce ${ }^{22}$, nourrit sans aucun doute l'absence totale de concertation, et même de communication concernant la question migratoire entre les autorités des deux pays. Celle-ci devient même un nouveau point de cristallisation des dissensions entre les deux États. Par exemple,

17. http://www.opex36o.com/2016/02/26/migrants-lotan-precise-le-mandat-de-son-operation-navale-en-mer-egee/ 18. http://www.ert.gr/gia-kindino-anthropistikis-katastrofis-stin-ellada-kani-logo-o-d-avramopoulos/ 19. Cyril Blondel, Guillaume Javourez, Stojanova Meri, « The European cross-border cooperation in the Balkan countries: Marking space and the multi-scalar production of locality ", in Nebi Bardhoshi, Gilles de Rapper, Pierre Sintès (dir.), Social Practices and Local Configurations in the Balkans, Tirana, UET, 2013, pp. 97-118. 20. Keith Brown, The Past in Question, Modern Macedonia and the Uncertainties of Nation, Princeton, Princeton University Press, 2003. 21. Athina Skoulariki, « La crise macédonienne (1991-1995) et la question des slavophones en Grèce ", in Balkanologie, vol. VII, n 1, 2003, pp. 137-146. 22. Anastasia Karakasidou, "Cultural illegitimacy in Greece : The slavo-macedonian "non"-minority ", in Richard Clogg (dir.), Minorities in Greece: Aspects of a Plural Society, Londres, C. Hurst and Co. Publishers Ltd, 2002, pp. 122-164. 
Lieux et frontières sur la « route des Balkans », décembre 2015

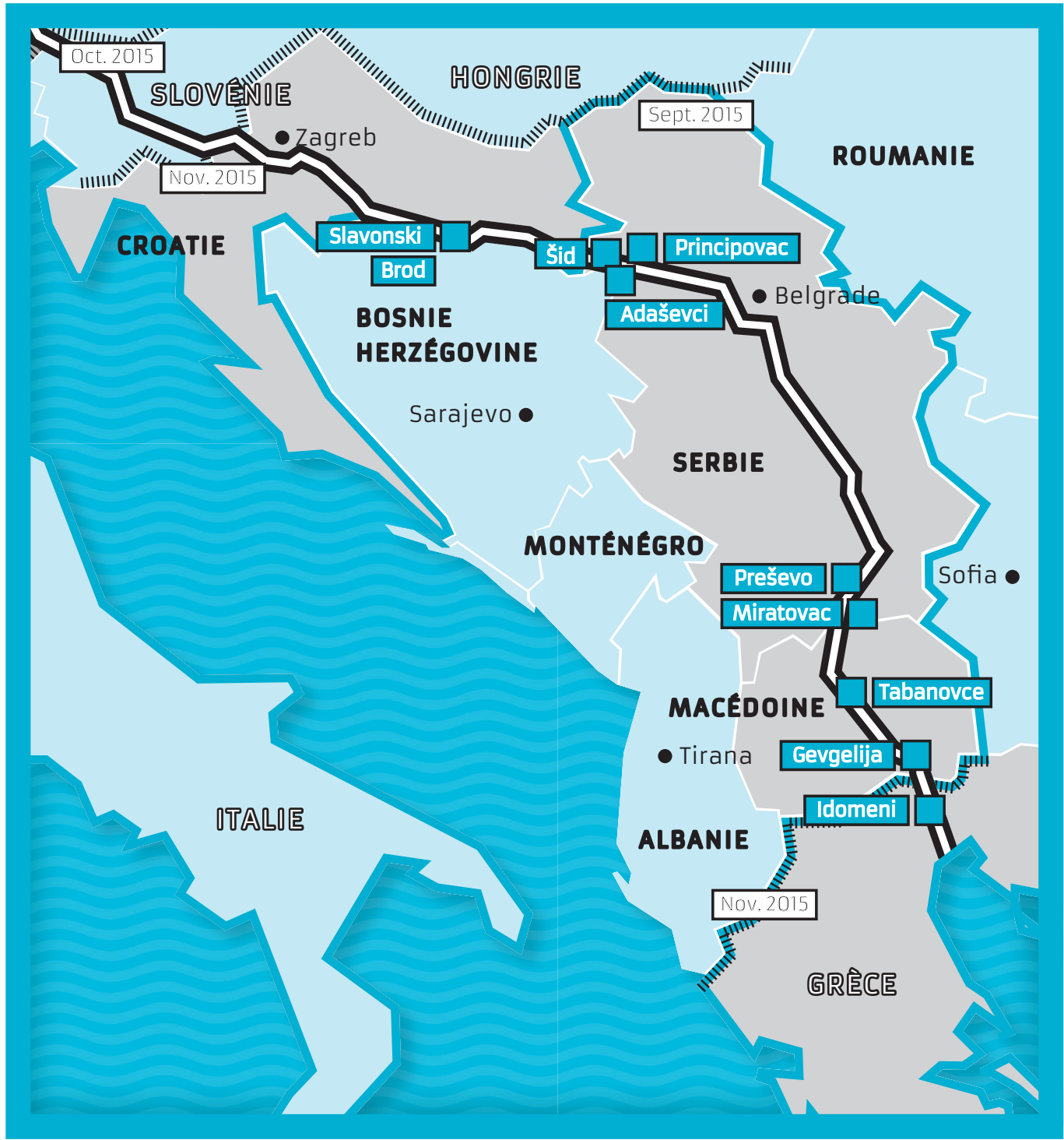

\begin{tabular}{|c|c|}
\hline IIIIIIIIIII & $\begin{array}{l}\text { Tracé du corridor } \\
\text { Murs : clôtures et grillages }\end{array}$ \\
\hline \begin{tabular}{|l|} 
Nov. 2015 \\
\end{tabular} & Date de fermeture \\
\hline & $\mathrm{F}$ rontières extérieures de l'UE \\
\hline - Tirana & Capitales \\
\hline Sidd & Camps de transit \\
\hline GGREిCE & Pays de l'espace Schengen \\
\hline ALBANIE & Autres pays de l'UE \\
\hline & Pays de la route des Balkans \\
\hline
\end{tabular}


la tension est montée mi-mars 2016 suite à la tentative d'un millier de migrants de traverser le cours d'eau Suva Reka pour entrer en territoire macédonien en contournant le blocage de la frontière à Idomeni. Leur arrestation et leur renvoi en Grèce ont provoqué des échanges acerbes entre les deux gouvernements ${ }^{23}$. Début avril 2016, une autre tentative d'incursion désespérée de migrants aux alentours d'Idomeni, au cours de laquelle les unités de la police macédonienne ont fait un usage massif de la force (grenades assourdissantes, gaz lacrymogène, balles en caoutchouc), a provoqué une nouvelle escalade verbale. Le Premier ministre grec, Alexis Tsipras, déclarait sans équivoque à ce propos : "C'est une honte pour la civilisation européenne " mais aussi «pour un pays qui prétend vouloir faire partie de cette civilisation ${ }^{24} "$, faisant directement planer une menace sur l'intégration possible de la République de Macédoine dans l'UE.

Mais la question migratoire n'est pas uniquement source de tensions pour les relations grécomacédoniennes. $\mathrm{Au}$ moment des incidents d'Idomeni le 13 mars, le Premier ministre bulgare, Boïko Borisov, s'indignait que les migrants connaissent des conditions de vie aussi déplorables dans les camps de rétentions grecs " malgré les 700 millions de levas versés par l'UE ${ }^{25}$ ». Une procédure de sécurisation commune Bulgarie/ République de Macédoine a d'ailleurs suivi afin de faire face à "la plus grande menace [qui] vient de la frontière grecque $e^{26} »$. Fin mai, les autorités bulgares ont même procédé à un premier renvoi vers la Grèce de 53 migrants afghans le jour même de leur arrivée en Bulgarie ${ }^{27}$, tout en affirmant, par la voix du premier secrétaire du ministère de l'Intérieur bulgare, que cette politique allait se poursuivre ${ }^{28}$. Le gouvernement bulgare se dit par ailleurs prêt à ériger une barrière sur une partie de sa frontière avec la Grèce, après avoir déjà fermé la frontière avec la Turquie. Cette même défiance se retrouve sur la frontière gréco-albanaise, puisque des policiers italiens sont venus y renforcer leurs collègues albanais depuis le 20 mars, en raison de la prévision d'un report incontrôlé des flux depuis la frontière gréco-macédonienne vers l'Italie, via l'Albanie. Même si ces reports ne sont pas avérés ${ }^{29}$, cette collaboration italo-albanaise est symptomatique de l'isolement de la Grèce dans ce dossier. La tendance est bien perçue depuis l'intérieur du pays, comme en témoignent les propos de Nikos Toskas, vice-ministre de l'Intérieur en charge du dossier migratoire, qui s'offusquait que des États, qui avaient pourtant envoyé plusieurs dizaines de milliers de migrants économiques vers la Grèce dans les années $1990^{30}$, refusent aujourd'hui les réfugiés en provenance du pays.

\section{Conclusion}

En quelques mois, la «route des Balkans» n'a doncpas survécu aux différentes actions des États souverains qui ont progressivement verrouillé leurs frontières après les décisions allemande et autrichienne de restreindre l'accueil des migrants. Plus d'une année après, la carte du Sud-Est européen présente de nombreux « murs » de grillages et de barbelés, de l'Autriche à la Bulgarie, de la Hongrie à la République de Macédoine. Si le «discours de l'urgence ${ }^{31}$ » qui caractérisait les années 2000 induisait une posture politique opportune pour négocier des positions à l'intérieur du cadre de gestion européen, la situation exceptionnelle qui s'engage à partir de l'été 2015 et sa qualification de "crise » par les différents

23. http://www.tovima.gr/politics/article/?aid=784421 24. http://www.skai.gr/news/politics/article/312184/tsipras-megalidropi-gia-ton-europaiko-politismo-i-stasi-tis-pgdm-stin-eidomeni/25. http://www.ambafrance-bg.org/Lundi-28-mars 26. http://www.voanews.com/content/bulgaria-build-fence-greece-border/3255227.html 27. "Un record en Europe » comme l'a affirmé lui-même le Premier ministre bulgare : http://greece.greekreporter. com/2016/05/30/bulgaria-returns-migrantswho-entered-illegally-from-greece/28. http://www.dailymail.co.uk/wires/reuters/article-3614358/Bulgaria-return-detainedAfghan-migrants-Greece.html 29. http://www.courrierdesbalkans.fr/le-fil-de-l-info/refugies-bloques-en-grece-konitza.html 30. Pierre Sintès, La raison du mouvement. Territoires et réseaux de migrants albanais en Grèce, Paris/Aix-en-Provence/Athènes, Karthala/Maison méditerranéenne des sciences de l'homme/École française d'Athènes, 2010. 31. Pierre Sintès, "Manœuvres politiques et discours de l'urgence : la frontière égéenne de la Grèce au cœur d'enjeux nationaux et européens », in Natalia Ribas-Mateos (dir.), El Rio Bravo Mediterráneo. Las Regiones Fronterizas en la época de la Globalización, Barcelone, Bellaterra, 2011, pp. 375-390. 
acteurs concernés ont imposé de nouvelles logiques dont la (re) mobilisation de discours belliqueux proprement nationaux est le signe le plus frappant. Une telle tendance renvoie à l'incapacité de l'acteur européen à dégager une perspective dans la gestion de ce dossier, et à son impossibilité structurelle à dépasser

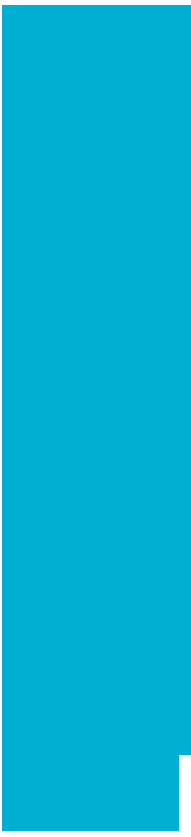
les cadres d'intervention nationaux. De façon paradoxale, c'est après le point culminant de la « crise » que les mécanismes communautaires ont repris la main sur les logiques nationales dans les Balkans. Avec la remise en fonction radicale depuis fin 2016 des mesures de renvoi vers le Sud-Est qui avaient été suspendues fin 2015 (tels les accords de Dublin et de réadmission), la région fait à nouveau office de zone-tampon de l'Europe ${ }^{32}$. Qu'on la considère comme une anomalie ou un révélateur des relations UE-Balkans dans le domaine migratoire, la parenthèse du corridor balkanique questionne avec force les capacités des cadres communautaires à répondre à ces situations d'urgences humanitaires.

À cet égard, cette " crise des migrants " peut plutôt être vue comme une véritable «crise des politiques migratoires $^{33}$ » communautaires. Pourtant, depuis plusieurs décennies, les lois migratoires européennes, loin de dissoudre les prérogatives nationales, sont exclusivement pensées à partir de préoccupations de la sécurité ou de la diplomatie, de la «culture des forces de l'ordre et du contrôle du territoire $^{34} »$. C'est pourquoi, si les institutions européennes ont bien connu une crise de leur fonctionnement, les logiques adoptées par les

Le camp fermé de Preševo (frontière serbo-macédonienne) conserve des traces de son ancienne fonction de camp de transit (2015-fev 2016) (c) Sarah Bachellerie, Sophie Clair.

différents États étaient loin d'être en rupture avec les principes de protection des frontières promues à l'échelle continentale depuis plusieurs décennies par l'UE. En conséquence, le fonctionnement irrégulier de la route officielle des Balkans et sa fermeture peuvent aussi être vus comme le résultat de ces politiques qui contiennent en elles-mêmes des contradictions avec les principes ou les valeurs communautaires. Le choix de ne pas enclencher de mécanismes humanitaires contraignants à l'échelle régionale et les conclusions du sommet européen de Bratislava de septembre 2016 en sont de parfaites illustrations. Sans répondre véritablement au drame humanitaire qui venait de se jouer plus au Sud ${ }^{35}$, cette rencontre a réaffirmé une fois de plus les priorités de protection des frontières extérieures, de lutte contre le terrorisme et de relance de la défense européenne comme réponse au défi migratoire. Marque de surenchère, d'inconscience ou de reniement, ce sont ces logiques qui se reflètent dans le repli des différents États... bonne application de ces mêmes principes de défense des frontières, mais entendues cette fois-ci à l'échelle de leur propre pays. 